\title{
A Discussion of the Current Limitations of Diabetes Etiology
}

\author{
Graham Wilfred Ewing \\ Mimex Montague Healthcare Limited, Mulberry House, Nottingham NG12 3TU, United Kingdom
}

*Corresponding author: Graham Wilfred Ewing, Mimex Montague Healthcare Limited, Mulberry House, 6 Vine Farm Close, Cotgrave, Nottingham NG12 3 TU, United Kingdom; Email: graham.ewing@mmhcl.co.uk; Tel: +44-115-9899618.

Received: May 04, 2020; Accepted: May 14, 2020; Published: June 12, 2020

\begin{abstract}
The immense amounts of research into diabetes reflects the need for a better understanding of what is diabetes (DM), how it can be measured, and how it can be treated and/or managed however despite the enormous amounts invested in diabetes research there is not unanimity from the research community on any of these matters. The issue which faces diabetes researchers is whether to continue with 'more of the same' lines of research or whether to strike out and seek more radical solutions.

It is clear that the biology of the pancreas plays a significant role; in particular the many factors which influence the genetic expression of insulin (type 1 diabetes) and the subsequent ability of insulin to react with its reactive substrate (type 2 diabetes); however there must be additional factors which are consistent with the laws of chemistry and physics which are not yet being seriously considered in the etiology of DM i.e. that the known biology of DM is only part of the complex etiology of DM.

The author raises a number of issues in this paper which address a number of inconsistencies in the screening and treatment of diabetes. He illustrates that type 1 and type 2 diabetes exist as comorbidities, that the onset of diabetes leads inevitably to the onset of a range of diabetic comorbidities, and that 'the regulation of blood glucose levels' exhibits the characteristics of a neurally regulated physiological system. Such observations have immense significance regarding how we screen for diabetes and how we treat diabetes and diabetic comorbidities.
\end{abstract}

\section{The Biology of Diabetes}

The first step to consider in the process of Diabetes (DM) is that of genetic expression in which a wide spectrum of genes, currently considered to be more than 60 genes [1], coherently interact in order to express pre-pro-insulin (type 1 diabetes) however the interpretation of such data is complicated when considering that the spectrum of genes which express pre-pro-insulin can differ [2] e.g. between different racial subtypes.

There are few instances, if any, where a single gene acts independently of all other genes in order to express a particular protein or where a single gene is considered to be responsible for a particular medical indication e.g. in the case of Rett's syndrome [3], which is considered to be widely attributable to a single mutation in the MECP2 gene, a more in-depth examination of the literature reveals a lack of certainty surrounding such a conclusion e.g.

- 'Mutations in a gene called MECP2 underlie almost all cases of classic Rett syndrome'

- 'Using a systematic gene screening approach, we have identified mutations in the gene (MECP2 ) encoding X-linked methyl-CpG-binding protein $2(\mathrm{MeCP} 2)$ as the cause of some cases of RTT'.

- 'In 5 of 21 sporadic patients, we found 3 de novo missense mutations in the region encoding the highly conserved methyl-binding domain (MBD) as well as a de novo frameshift and a de novo nonsense mutation, both of which disrupt the transcription repression domain (TRD)'

- 'In two affected half-sisters of a RTT family, we found segregation of an additional missense mutation not detected in their obligate carrier mother'

This indicates that there is conceivably a phenomenon of greater significance than gene chemistry but which nevertheless involves gene chemistry. It can only be explained if our DNA and genes are continually seeking out more stable states, in a best-fit manner i.e. changing between different conformational and energetic states [4] in order to find the most stable state.

It conceivably explains why therapies, which were designed to influence the genetic profile in order to be effective in their specific areas of application (viruses, virus-like particles and/or and vaccines), are associated with predisposition to type 1 diabetes [5].

1. It is often overlooked that genetic expression is a chemical reaction in which various components e.g. the genetic spectrum, transcriptases and minerals; influence the rate and extent to which pre-pro-insulin is expressed however transcriptases are often dependent upon magnesium and zinc for their structure, function and reactivity. This is significant in DM because the level of essential minerals and/or their reactivity are dependent upon the prevailing intercellular $\mathrm{pH}$ 
therefore a deficit of minerals, in particular magnesium and zinc which is commonly observed in the diabetic [6], must influence the structure and/or reactivity of the enzyme and hence the extent of expression of pre-pro-insulin.

In general, the conversion of pre-pro-insulin to pro-insulin is considered to proceed without any significant hindrances however it should be emphasised that the conversion of pre-pro-insulin is dependent upon the availability and reactivity of signal peptidases and hence is likely to be influenced by the prevailing intercellular $\mathrm{pH}$ and levels of minerals and cofactors although the extent to which this occurs is uncertain.

2. Similarly the conversion of pro-insulin to insulin must also be dependent upon the prevailing reaction kinetics. Moreover, it is encoded by the INS gene [7] so any changes of gene structure [1] due to the influence of viruses and/or viruslike particles, the presence of mutant alleles, the influence of epigenetic components, the action of reactive oxygen species upon gene structure, alterations of gene conformation and energetics, and the prevailing reaction conditions e.g. $\mathrm{pH}$ and mineral levels; must also influence this process to some extent.

3. It is considered that because pro-insulin has a longer halflife than insulin (if not insulin levels would be in a perpetual deficit) and that this accounts for $5-30 \%$ of the insulin-like structures in the blood however there does not appear to be any significant explanations for this broad range of proinsulin levels [8]. This may be significant, especially so if we consider that only one of the many insulin-like structures insulin monomer - will react with its receptor protein IRP2.

4. Pro-insulin is converted to insulin by endopeptidases (PC1 \& PC2) and Carboxypeptidase $\mathrm{E}$ which function at rates determined by their unique chemical properties and the corresponding reaction kinetics involving intercellular $\mathrm{pH}$, levels of minerals, etc.

5. Insulin is a very large and highly reactive protein comprises 51 amino-acids. It exists as a dimer (particularly so when complexed as the zinc-hexamer), comprising an A chain and a B chain which are connected by disulphide bonds but reacts as the monomer. Its chemical properties are that it exists as a coiled moiety, is a polar entity with $-\mathrm{NH} 2$ and $-\mathrm{COOH}$ groups at its extremities, is water soluble, and highly reactive (half-life of ca 3 minutes) so if the $\mathrm{pH}$ is not maintained at the appropriate level this will influence insulin conformation, energetic state and half-life.

6. The relatively slow genetic expression/production of insulin, conceivably because it is such a large and highly reactive molecule, requires that insulin is stored in the pancreas as a zinc-hexamer; awaiting metabolic signals and vagal nerve stimulation [9] to be exocytosed from the cell into the circulation; however under pathological conditions i.e. elevated levels of intercellular acidity, reduced levels of essential minerals, alters the redox state and/or bioavailability and levels of transition metals. As a result insulin becomes less coiled and circulates as the monomer rather than as the more stable dimer or the hexamer.

It provides a mechanism whereby zinc availability and redox mechanism are linked. Zinc is transported by albumin and transferrin however transferrin also transports iron which reduces the absorption of zinc, and vice versa. Zinc and copper also have an antagonistic relationship. This is consistent with differing levels of intercellular acidity in which there is a biodynamic relationship between essential minerals and the transition minerals at $\mathrm{pH} 7.35$. When intercellular $\mathrm{pH}$ decreases to $\mathrm{pH}$ of more typically 6.75 the levels of essential minerals such as zinc and magnesium decline whilst the levels of transition metals such as iron, aluminium, copper increase. It explains why most diabetic patients are magnesium and zinc deficient [10-12].

Moreover [13] insulin levels vary throughout the day, from $<100 \mathrm{pmol} / \mathrm{l}$ to $>800 \mathrm{pmol} / \mathrm{l}$, and in particular (i) following a meal and (ii) in a 3-15 minute oscillating manner i.e. indicatively over a 3-6 minute period for those who are normally healthy and over a 6-15 minute period for the diabetic. Under acidic conditions insulin becomes less coiled and less reactive (the phenomena of 'proteinresistance' is common to insulin, leptin, ghrelin and perhaps also in the 'folding' of other proteins), the availability of zinc hexamer in the islets declines [14] under acidic conditions, and insulin is supplied over a longer period and/or in a less reactive form.

Pathological onset of DM under acidic conditions (in general, DM does not occur in patients with relatively neutral intercellular $\mathrm{pH})$, is accompanied by the glycation of proteins $[15,16]$ e.g. of insulin, albumin, LDL-Cholesterol, haemoglobin, fibrinogen, immunoglobulin(s), etc. This is indicative of free radical reactions which arise from increased acidity, increased levels of transition minerals [17], and a suitable substrate (glucose). It is confirmed by noting that anti-oxidants [18] influence such oxidative processes and have a positive influence upon DM.

In addition the reaction of insulin with its receptor protein IRP2 is a magnesium dependent reaction and, as outlined, the supply of magnesium is dependent upon prevailing levels of intercellular $\mathrm{pH}$.

7. That the regulation of intercellular $\mathrm{pH}$ is so immensely significant, if not obvious from the above, is further confirmed when considering the role of metformin which is eliminated from the body almost completely unmetabolised therefore its metabolic effect is not pharmacological. A closer examination of metformin's chemical structure reveals that it exhibits the structure, and hence the function, of a biological buffer [19] which maintains intercellular $\mathrm{pH}$ at a level of indicatively 6.75-6.95.

Accordingly if applied to the diabetic patient it will have the effect of reducing intercellular $\mathrm{pH}$ in patients with a lower intercellular $\mathrm{pH}$ i.e. the severely diabetic and/or obese patient with intercellular $\mathrm{pH}$ of indicatively 6.25-6.75. This should have the effect of reducing the severity of their diabetic symptoms however it will not be a substitute for weight-reduction measures. For patients who are pre-diabetic, who have been prescribed metformin, it will likely have the effect of lowering their intercellular $\mathrm{pH}$ and enhancing their diabetic symptoms. 
8. The expression of insulin and the reaction of insulin with its receptor protein are sequential processes. If they were parallel processes there would be a selection of one of the two processes - the 'either or' scenario - but this is clearly not the case. The genetic expression of a protein is followed by the reaction of the expressed protein with its reactive substrate. The two processes occur in sequence so type 1DM (genotype) and type 2DM (phenotype) are comorbidities in which there can be several different states e.g.

(i) low levels of type1DM and absence of type 2DM,

(ii) low levels of type 1DM and low levels of type 2DM (diagnosis: prediabetes),

(iii) low levels of type 1DM and high levels of type 2DM (type 2 diabetes),

(iv) high level of type 1DM and absence of type 2DM (diagnosis: type 1 diabetes),

(v) high level of type 1DM and low/moderate level of type 2DM (diagnosis: type 1DM)

(vi) high level of type 1DM and high level of type 2DM (diagnosis: type 1 and type $2 \mathrm{DM}$ )

It is not a case of whether the patient has either type 1DM or type 2DM. Both can and do occur as comorbidities as outlined in (ii)-(vi).

9. The GLUT-4 receptor which converts glucose into energy is located in the smooth muscle. Moreover this reaction is a chromium-dependent reaction in which the prevailing redox states of chromium are influenced by the prevailing intercellular $\mathrm{pH}$ i.e. the intercellular $\mathrm{pH}$, in combination with the quality and quantity of smooth muscle (physical fitness) influences the ability to metabolise blood glucose.

\section{Measuring Diabetes}

The onset of pathological conditions, as outlined, leads to the production of mainly excess blood glucose and elevated levels of advanced glycation end-products, which are manifest as glycated proteins and other lipids, and which subsequently alter blood viscosity and thereby influence the function of the heart, kidneys, etc. Nevertheless, and as outlined in 1.10 above, this hyperglycaemia which is characteristic of type $1 \mathrm{DM}$, is only one aspect of diabetes. Hypoglycaemia can be encountered due to suppression of the genetic expression of insulin. Nevertheless the characteristics of diabetes, in particular of unstable levels of blood glucose, can occur due to pathological onset in other organs (mainly the endocrine glands) and physiological systems e.g. in the case of hysterectomy.

The glycated protein $\mathrm{HbA1c}$ is used as a measure of type 2 diabetes however the validity of the test is often questioned, perhaps with good reason [20]. The processes responsible for type 1 and type 2 occur simultaneously i.e. they are comorbidities; thereby explaining to some extent the misdiagnoses when using the HbAlc test [21, 22], but the test only measures the glycation process.

The A1c form of glycated haemoglobin A was selected because it was considered to be the most prominent of the various forms of glycated haemoglobin however it is likely, perhaps inevitable, that altered reaction conditions will lead to increased/decreased levels of the different glycated haemoglobin isomers and a reduction/increase of HbAlc.

\section{'the measurement of $\mathrm{HbA}_{1 \mathrm{C}}$ is likely not a comprehensive indicator of $\mathrm{HbA}$ glycation' [23]}

Also, it is assumed that the ratio of haemoglobin A vs other proteins does not vary and hence that the test would be an accurate measure of the glycation process however the level of haemoglobin varies widely [24]. Test outcomes are influenced by many variables including light, $\mathrm{pH}$, levels of minerals, $\mathrm{RBC}$ and/or haemoglobin levels, and other factors; and finally haemoglobin A does not play a role in the etiology of diabetes! Accordingly, the scope for misdiagnosis is immense. The possibility of inaccurate test results is inevitable, especially so in cases of non-pancreatic diabetes in which the problems of blood glucose regulation are not caused by the aforementioned pathological processes which are commonly attributed to diabetes but instead to pathological emergence in other organs and physiological systems [25].

All chemical reactions are accompanied by the absorption and emission of energy

i.e., by the emission of biophotons which create the phenomena of auto fluorescence and/or bioluminescence in blood [26]. The emission of biophotons $[27,28]$, as proteins decay from their reactive state to their base state, is particularly evident in the retina of diabetics as it alters blue-yellow colour perception [29] and serves as a measure of type 1 and type 2 diabetes in a cognitive test.

This occurs because the eye responds to as little as $7^{\star} 10^{\wedge} 2$ bio photons per second. The retina focusses this bioluminescence which can be measured in a cognitive test and used as a precise, digital measure of pathological onset and progression in all medical conditions which are accompanied by changes of colour perception and brain function i.e. across the complete medical spectrum. The company Bio Astral [28], supported by significant UK government grants prior to its demise, was researching advances in space-science imaging technology i.e. to measure the release of bio photons from blood (quote: 'Our STJ cryogenic detector is 1000 times more powerful at detecting fluorescence in biological assays than current technology, and is unique in giving the colours of photons detected without requiring filters, gratings or other techniques').

Accordingly a technique which measures the intensity and colour of biophotons has significant potential as a measure of diabetes which is relatively free from side-effects, errors and misdiagnoses.

\section{The Neurology of Type 2 Diabetes}

There are two fundamental avenues which influence the autonomic nervous system: (i) via sensory intake (vision, hearing, smell, taste and touch) and (ii) via the visceral organs.

As outlined in this text, genetic and non-genetic (phenotype) changes lead to the onset of type 1 and type 2 diabetes. It is commonly 
considered that genetic changes occur due to the effect of gene-altering moieties e.g. as viruses; however this text illustrates that genetic changes occur due (i) to the influence of virus-like particles (vaccines) and (ii) increased levels of intercellular $\mathrm{pH}$ which accompany stress and generate ROS which have a degenerative effect upon the genetic profile.

Moreover stress (either as psychological stress or as psychophysiological stress i.e. excess weight), which is experienced via the sensory organs and brain, is an acidifying process which increases cortisol levels and influences appetite [30] thereby illustrating the link between molecular biology and sense perception - influencing perception of colour and appetite.

So type 2 Diabetes is a neurological condition which arises because the brain is unable to maintain the calorific balance between appetite, the intake of calories through food and drink, and the expenditure of energy.

The regulation of blood glucose exhibits the characteristics of a neurally regulated physiological system whereby blood glucose levels are maintained between higher and lower levels hence the use of the terms 'hyper'-glycaemia and 'hypo'-glycaemia.

The regulation of blood glucose is just one of the 13 physiological systems. The other systems, identified by Grakov IG, are: blood pressure, blood volume, blood cell content, respiration, the consumption of food and drink, the elimination of fluids, intercellular $\mathrm{pH}$, body temperature, posture, osmotic pressure, sexual function and sleep. Each performs an indispensable physiological function which, in most cases, and if not sustained within specific limits, leads to our demise. Each physiological system involves the brain and the endocrine glands.

Moreover, and as outlined earlier, the regulation of blood glucose involves the vagus nerve [9] which stimulates the pulsed release of insulin [13] by the pancreas.

Accordingly pathological onset in organs within this physiological system (network of organs) or in adjacent physiological systems will influence blood glucose levels. It explains the phenomena of nonpancreatic diabetes in which the patient(s) have problems regulating their blood glucose and yet their pancreas' functions [31] normally e.g. due to endocrine dysfunction, hysterectomy, etc.

The most significant physiological systems [31] which influence the regulation of blood glucose level are sleep, $\mathrm{pH}$ [32], what we consume and excrete, and body temperature.

Each physiological system can be neurally regulated.

It is a problem for biomedicine to explain, using a solely chemical mechanism, how the different organ networks act in an apparently coherent manner. In 2013 the Human Brain Project was established to explore the issues. It was tasked by the EC to achieve three fundamental objectives: (i) to understand what the brain does and how it does it; (ii) to use this knowledge to develop a new generation of cognitive diagnostic test, and (iii) to understand and adapt with therapeutic effect the multilevel nature of brain function. Specific emphasis was placed upon screening the complex pathological coordinates of Alzheimer's disease.
Ewing recognised that these fundamental objectives had been completed by Grakov in the period 1981/2-1997-2006 and were incorporated into the Strannik software. Papers followed which compared the two different techniques [33,34]; identified fundamental limitations of the techniques being deployed to achieve such objectives $[34,35]$, in particular the reliance upon 'big data'; determined that the brain acts as a neuromodulator [36] of the autonomic nervous system; that Grakov had developed a screening test which can screen for the complex pathological correlates of diabetes and diabetic comorbidities including Alzheimer's Disease [37-42]; and had understood how this led to an understanding of the multilevel nature of brain function in particular of the mechanism which regulated the stability of the autonomic nervous system and the coherent function of the physiological systems [43].

The specific origins of such a technique can be traced back for over 100 years however it is only in the last 10-20 years that significant progress is being made towards the development of the envisaged new generation of neuromodulation therapy [44-46] which has the potential to improve therapeutic outcomes in [47] a wide range of medical indications [48-55] including multiple sclerosis, Parkinsonism, Alzheimer's Disease, Migraine, regulation of menstruation, etc.

\section{Discussion}

The evidence assembled in this short paper illustrates that type 1 and type 2 diabetes are comorbidities and that the regulation of blood glucose levels' exhibits the characteristics of a neurally regulated physiological system and involves the process of neuroregulation [56, 57] i.e. the relationship between sense perception, brain function, the autonomic nervous system (the stress response) and physiological systems, and cellular and molecular biology (as genotype and phenotype).

Moreover the evidence for such a conclusion, and its relevance to how we screen or treat diabetes is already evident in various diabetes papers which illustrate that (i) changes of blue-yellow colour perception accompany the onset and progression of diabetes, (ii) that this phenomena is not unique to diabetes but can be used to screen for an extensive range of pathologies [38] including diabetic comorbidities, (iii) that the phenomena is associated with the emission of biophotons, (iv) that the vagus nerve is involved in diabetes etiology, (v) that insulin is released by the pancreas in a pulsatile manner which is indicative of 'control circuitry', and (vi) that knowledge of this 'neuro-regulatory mechanism' can be used to treat patients with a wide range of pathological indications [39] including type 1 and type 2 diabetes and related diabetic comorbidities e.g. by reducing exposure to stress $[30,58]$.

The future development of neurological knowledge, in particular of how the brain works and what it does, is leading to the development of a new generation of diagnostic and therapeutic technologies as envisaged by the EC's Human Brain Project although perhaps such technologies [59] have not emanated from Human Brain Project research. 


\section{References}

1. Bakay M, Pandey R, Hakonarson H (2013) Genes involved in type 1 diabetes: an update. Genes (Basel) 4(3): 499-521. [crossref]

2. Ewing GW (2017) The Interpretation of Genetic Data - Considering the Effect of Changes to Gene Conformation -- If the facts don't support the theory, change the theory - how does this contribute to understanding Diabetes? J Genet Disor Genet Rep 6: 1-4.

3. Amir R, Van den Veyver I, Wan M, Tran C, Francke U et al. (1999) Rett syndrome is caused by mutations in X-linked MECP2, encoding methyl-CpG-binding protein. Nat Genet. 23: 185-188.

4. Bodhini D, Radha V, Ghosh S, Majumder PP, Mohan V (2011) Lack of association of PTPN1 gene polymorphisms with type 2 diabetes in south Indians. J Genet 90: 323-326. [crossref]

5. Tuomilehto J, Rewers M, Reunanen A, Lounamaa P, Lounamaa R et al. (1991) Increasing Trend in type 1 (insulin-dependent) diabetes mellitus in childhood in Finland.Analysis of age, calendar time, and birth cohort effects during 1965-1984. Diabetologia 34: 282-287. [crossref]

6. Gunther $\mathrm{T}$ (2010) The biochemical function of $\mathrm{Mg} 2+$ in insulin secretion, insulin signal transduction and insulin resistance. Magnesium Research 23: 5-18. [crossref]

7. INS insulin [ Homo sapiens (human) ] [crossref]

8. Interpretation for 80908 Proinsulin, Plasma. www.mayomedicallaboratories.com. 2017-03-09.

9. Meyers EE, Kronemberger A, Lira V, Rahmouni K, Stauss HM. (2016) Contrasting effects of afferent and efferent vagal nerve stimulation on insulin secretion and blood glucose regulation. Physiol.Rep 4: 12718.

10. Maret W, Krezel A (2007) Cellular zinc and redox buffering capacity of metallothionein/thionein in health and disease. Mol Med 13: 371-375.

11. Rink L, Gabriel P (2000) Zinc and the immune system. Proc Nutr Soc 59: 541-552. [crossref]

12. Brandão-Lima PN, Carvalho GB, Santos RKF, Santos BDC, Vasconcelos NLD et al. (2018) Intakes of Zinc, Potassium, Calcium, and Magnesium of Individuals with Type 2 Diabetes Mellitus and the Relationship with Glycemic Control. Nutrients 10: 1948.

13. Hellman B, Gylfe E, Grapengiesser E, Dansk H, Salehi A (2007) Insulin oscillationsclinically important rhythm. Antidiabetics should increase the pulsative component of the insulin release. Läkartidningen 104: 2236-2239. [crossref]

14. Wijesekara N, Chimienti F, Wheeler MB (2009) Zinc, a regulator of islet function and glucose homeostasis. Diabetes, Obesity and Metabolism 11: 202-214. [crossref]

15. Singh VP, Bali A, Singh N, Jaggi AS (2014) Advanced Glycation End Products and Diabetic Complications. Korean J Physiol Pharmacol 18: 1-14. [crossref]

16. Wolff SP (1993) Diabetes mellitus and free radicals: Free radicals, transition metals and oxidative stress in the aetiology of diabetes mellitus and complications. British Medical Bulletin 49: 642-652. [crossref]

17. Aust SD, Morehouse LA, Thomas CE (1985) Role of metals in oxygen radical reactions. J Free Radic Biol Med 1: 3-25. [crossref]

18. Maritim AC, Sanders RA, Watkinss JB 3rd (2003) Diabetes, oxidative stress, and antioxidants: a review. J Biochem Mol Toxicol 17: 24-38. [crossref]

19. Ewing GW (2018) Is Metformin a Drug or a Buffer and Why is this Significant? Further evidence that the brain Regulates the Autonomic Nervous System, in particular prevailing levels of Intercellular pH. Endocrinology, Diabetes and Metabolism Journal 2: 1-9.

20. McDonald TJ, Warren R (2014) Diagnostic confusion? Repeat HbAlc for the diagnosis of diabetes. Diabetes Care 37: 135-136.

21. Cheol Bae J, Sunghwan S, Sang-Man J, Se won K, Kyu Yeon H, et al. (2014) Hemoglobin Alc values are affected by haemoglobin level and gender in non-anaemic Koreans. Journal of Diabetes Investigation 5: 60-65. [crossref]

22. Hegde SN, Srikousthubha MS, Anupama YJ, Rathnakar R, et al. (2018) A case of undetectable glycated haemoglobin (HbAlc). QJM: An International Journal of Medicine 111: 567-568.

23. Clark SLD, Santin AE, Bryant PA, Holman RW, Rodnick KJ (2013) The initial noncovalent binding of glucose to human haemoglobin in nonenzymatic glycation. Glycobiology 23: 1250-1259. [crossref]
24. Ebben JP, Gilbertson DT, Foley RN, Collins AJ (2006) Haemoglobin level variability: Associations with comorbidity, intercurrent events, and hospitalizations. Clin J Am Soc Nephrol 1: 1205-1210. [crossref]

25. Ewing GW, Ewing EN (2008) NeuroRegulation of the Physiological Systems by the Autonomic Nervous System - their relationship to Insulin Resistance and Metabolic Syndrome. Biogenic Amines 22: 208-239.

26. Ewing GW, Parvez SH, Grakov IG (2011) Further Observations on Visual Perception: the influence of pathologies upon the absorption of light and emission of bioluminescence. The Open Systems Biology Journal 4: 1-7.

27. Ewing GW, Parvez SH, Grakov IG (2011) Further Observations on Visual Perception: the influence of pathologies upon the absorption of light and emission of bioluminescence. The Open Systems Biology Journal 4: 1-7.

28. https://www.le.ac.uk/biology/phh4/bioastral/home.htm

29. Radwan TM, Ghoneim EM, Ghobashy WA, Orma Ahmed A (2015) Assessment of Color Vision in Diabetic Patients. International Journal of Ophthalmic Research 1: 19-23.

30. Razzoli M, Pearson C, Crow S, Bartolomucci A (2017) Stress, overeating, and obesity: Insights from human studies and preclinical models. Neurosci Biobehav Rev 76: 154162. [crossref]

31. Ewing GW (2013) The 'Biology of Systems' or the 'Systems of Biology': Looking at Diabetes from the Systemic Perspective. International Journal of Systems Biology 4: 45-56.

32. Ewing GW (2012) The Regulation of $\mathrm{pH}$ is a Physiological System. Increased Acidity alters Protein Conformation and Cell Morphology and is a Significant Factor in the onset of Diabetes and other common pathologies. The Open Systems Biology Journal 5: $1-12$.

33. Ewing GW, Grakov IG (2015) A Comparison of the Aims and Objectives of the Human Brain Project with Grakov's Mathematical Model of the Autonomic Nervous System (Strannik Technology). Enliven: Neurol. Neurotech 1: 002.

34. Ewing GW (2015) Back to Basics: Limitations of Research influencing the Human Brain Project. Comput.Sci.Syst.Biol 8:6:322-326.

35. Ewing GW (2017) The Limitations of Big Data. The Limitations of Big Data in Healthcare. MOJ Proteomics Bioinform 5: 00152.

36. Ewing G (2016) What is the function of the Brain? What does it do and how does it do it? It functions as a Neuroregulator, which continuously regulates the Autonomic Nervous System and Physiological Systems, and enables us to Recognise that Sleep Exhibits the Characteristics of a Neurally Regulated Physiological System. J Neurol Psychol 4: 9.

37. Ewing GW (2016) The Use of Strannik Virtual Scanning as a Modality for the Earliest Screening of the Pathological Correlates of Alzheimer's Disease. Human Frontier Science Program (HFSP) Journal 10: 2-20.

38. Ewing GW (2018) A Different Perspective on Diabetes \& Obesity - what it is and how it can be measured. Case Reports in Clinical Medicine 7: 269-287.

39. Ewing GW, Parvez SH (2019) Diabetes is a Complex Neurological, Multisystemic, Multipathological and Polygenomic disorder : Use of Strannik Software as an Effective Modality to Illustrate its Complexity. J Neurol Psychol 7: 14.

40. Ewing GW, Ewing EN (2009) Computer Diagnosis in Cardiology. N Am J Med Sci 1: 152-159.

41. Ewing GW (2016) The Use of Strannik Virtual Scanning as a Modality for the Earliest Screening of the Pathological Correlates of Alzheimer's Disease. Human Frontier Science Program (HFSP) Journal 10: 2-20.

42. Ewing GW (2019) How Disruptive Solutions Could Improve the Prevailing Healthcare Paradigm. A Novel Perspective Regarding the Treatment of Mental Health Conditions: the Strannik Mathematical Model. J Clin Cases Rep 3: 89-114.

43. Ewing GW (2010) A Theoretical Framework for Photosensitivity: Evidence of Systemic Regulation. Journal of Computer Science and System Biology 2: 287-297.

44. Ewing GW (2010) There is a need for an Alternative or Modified Medical Paradigm involving an understanding of the nature and significance of the Physiological Systems. N Am J Med Sci 2: 1-6.

45. Ewing GW (2013) Virtual Scanning: a New Medical Paradigm? Journal of Computer Science and Systems Biology 6: 93-98. 
46. Ewing GW (2016) The Future of Medicine: Biomedicine or Neuroscience? Insights in Biomedicine 1: 14-17.

47. Kelley R, Flouty O, Emmons EB, Youngcho Kim, Johnathan Kingyonet al. (2017) A human prefrontal-subthalamic circuit for cognitive control. Brain 141: 205-216. [crossref]

48. Abboud H, Hill E, Siddiqui J, Alessandro Serra, Benjamin Walter (2018) Neuromodulation in multiple sclerosis. Mult Scler X 23: 1663-1676. [crossref]

49. Gaede G, Tiede M, Lorenz I (2018) Safety and preliminary efficacy of deep transcranial magnetic stimulation in MS-related fatigue. Neurology: Neuroimmunology \& Neuroinflammation 5: 423 .

50. Lv Q, Du A, Wei W, Li Y, Liu G, Wang X-P (2018) Deep Brain Stimulation: A Potential Treatment for Dementia in Alzheimer's Disease (AD) and Parkinson's Disease Dementia (PDD). Front Neurosci 12:360. [crossref]

51. Martorell AJ, Paulson AL, Suk H-J, Fatema Abdurrob, Gabrielle T Drummondet al. (2019) Multi-sensory Gamma Stimulation Ameliorates Alzheimer's-Associated Pathology and Improves Cognition. Cell 77: 256-271. [crossref]

52. Iaccarino HF, Singer AC, Martorell AJ, et al. (2016) Gamma frequency entrainment attenuates amyloid load and modifies microglia. Nature 540: 230-235.
53. Luijmes RE, Pouwels S, Boonman J (2016) The effectiveness of neurofeedback on cognitive functioning in patients with Alzheimer's disease: Preliminary results. Neurophysiol Clin 46:179-187. [crossref]

54. Noton D (2000) Migraine and photic stimulation: report on a survey of migraineurs using flickering light therapy. Complement. Ther Nurs Midwifery 6: 138-142. [crossref]

55. Anderson DJ, Legg NJ, Ridout DA (1997) Preliminary trial of photic stimulation for premenstrual syndrome. J Obstet Gynaecol 17: 76-79. [crossref]

56. Ewing GW, Parvez SH 2011 (2011) Mathematical Modeling the Systemic Regulation of Blood Glucose: 'a top-down' Systems Biology Approach. NeuroEndocrine Letters 32: 371-379. [crossref]

57. Ewing GW (2015) A Framework for a Mathematical Model of the Autonomic Nervous System and Physiological Systems using the NeuroRegulation of Blood Glucose as an Example. J Comput.Sci Syst Biol 8: 59-73.

58. Martin Cosgrove, Martin Cosgrove (2004) Do stressful life events cause type 1 diabetes? Occupational Medicine 54: 250-254.

59. Grakov IG Strannik Diagnostic and Treatment System: a Virtual Scanner for the Health Service. Minutes of Meeting No. 11 of the Praesidium of the Siberian of the Academy of Medical Sciences of the USSR (AMN) held in Novosibirsk 4 December 1985. 